The latitude of the cosmic-ray knee appears to shift during the solar cycle $\theta^{5}$ and simultaneous determinations in the two hemispheres are desirable in order to choose the most suitable magnetic coordinates. Observers in the northern hemisphere have reported knee positions in the neighbourhood of $53^{\circ} \mathbf{N}$. geomagnetic latitude and it is suggested that conventional geomagnetic co-ordinates are to be preferred at these latitudes since they preserve the symmetry between the two hemispheres. This view receives support from the work of V. D. Hopper, Jean E. Laby and Y. K. Lim (private communication) who measured directly the critical energies for heavy nuclei at Melbourne using water-loaded emulsions and find agreement with the Stormer theory using conventional geomagnetic co-ordinates rather than rotated co-ordinates.

This work was made possible by the generosity of the Royal Australian Air Force in providing the aircraft and accurate navigational information. Financial support was received in the form of a grant from the Australian Academy of Science.

J. R. Storey

A. G. Fenton

I. G. MCCRACKEN

Physics Department,

University of Tasmania,

Hobart, Tasmania. Feb. 18.

1 Simpson, J. A., Fenton, K. B., Katzman, J., and Rose, D. C., Phys. Rev., $102,1648(1956)$.

'See, for example, Waddington, C. J., Nuovo Cim., 3, 930 (1956).

$s$ British Admiralty Magnetic Charts (1955). - Rothwell, P. and Quenby, J., Proceedings of the Varenna Conference,
June 1957.

'Meyer, P., and Simpson, J. A., Phys. Rev., 99, 1517 (1955).

\section{Auroral Absorption of 18-Mc./s. Cosmic Radio Waves on February II, 1958}

ON the night of February 10-11, 1958, a spectacular auroral display was widely observed in the United States. Fig. 1 shows the effect of this aurora on 18-Mc./s. cosmic noise from the sky, as observed at the Sampson Station of this Observatory (lat. $42^{\circ}$ $47^{\prime} \mathrm{N}$., long. $73^{\circ} 27^{\prime} \mathrm{W}$.). The vertical scale indicates approximate relative absorption of cosmic noise in decibels as received. The horizontal scale gives Universal Time on February 11. The dotted line represents the record of the following night, February 12 , which itself shows at 7 hr. U.T. a characteristic dip which we consider to be associated with auroral activity. The peak at 0915 is a calibration mark. We note that the time marks, which are produced every $10 \mathrm{~min}$. from $W W V$ signals at $5 \mathrm{Mc} . / \mathrm{s}$., fade out at 0250 for the remainder of the night.

This record was obtained with an apparatus designed to detect sudden absorptions of cosmic noise as indirect indicators of solar flares. The antenna pattern covers most of the sky, with the gain greatest in the vertical direction. The receiver output depends on the minimum noise incident on the antenna as the frequency is swept over a range of $30 \mathrm{kc} . / \mathrm{s}$., to avoid interference by radio stations. The system is described briefly in Astron. J., 62, 243 (1957); a fuller description will be given in a forthcoming publication of this Observatory.

\section{Observatory of Rensselaer Polytechnic Institute, Troy, New York.} March 3.

\section{Calculation of the Life-time of a Satellite}

Three recent discussions of the effect of friction on a satellite ${ }^{1-3}$ have been based on the assumptions (1) that the atmosphere is spherically symmetrical, (2) that $S C_{D}$ is constant, where $C_{D}$ is the drag coefficient and $S$ the cross-sectional area perpendicular to the direction of motion, and (3) that the density of the atmosphere is exponential over the heights within which the perigee lies. The convenience of the third assumption is that the actual values of the density and the scale-height are not required, the mere constancy of the scale-height being sufficient. On the other hand, an elaboration of (3) requires a knowledge of the density distribution and is likely to lead to less rather than greater accuracy. The failure of (2) will presumably be a consequence of rotation of the satellite and will introduce periodic rather than progressive variations in the orbit. It seems that greater accuracy should be sought in correction for the lack of spherical symmetry of the atmosphere; this can be done with only a minor modification of the analysis of Scott ${ }^{2}$.

The effect of the rotation and oblateness of the Earth is to introduce a first-order correction to the density distribution in the form :

$$
\rho=\rho_{0} \exp \left\{-\frac{z}{H}-\frac{\varepsilon R \sin ^{2} \alpha \cos ^{2} \theta}{H}\right\}
$$

where $p_{0}$ is a constant, $H$ the atmospheric scale-height, $\varepsilon$ the ellipticity of the Earth, $z$ the height above the surface of the Earth, $R$ the radius of the Earth, $\alpha$ the inclination of the orbit to the plane of the equator and $\theta$ the angular distance from perigee in the plane of the orbit.

If we write the equation of the orbit in the form :

$$
r=p+A(1-\cos \theta)
$$

and carry through the analysis in the form used by Scott ${ }^{2}$, we find that :

$$
\begin{gathered}
\dot{p}=-C^{\prime} \exp \left(-\frac{p}{H}\right)\left(\frac{A^{\prime}}{H}\right)^{-3 / 2} \\
\dot{A}^{\prime}=-2 C \exp \left(-\frac{p}{H}\right)\left(\frac{A^{\prime}}{H}\right)^{-1 / 2}
\end{gathered}
$$

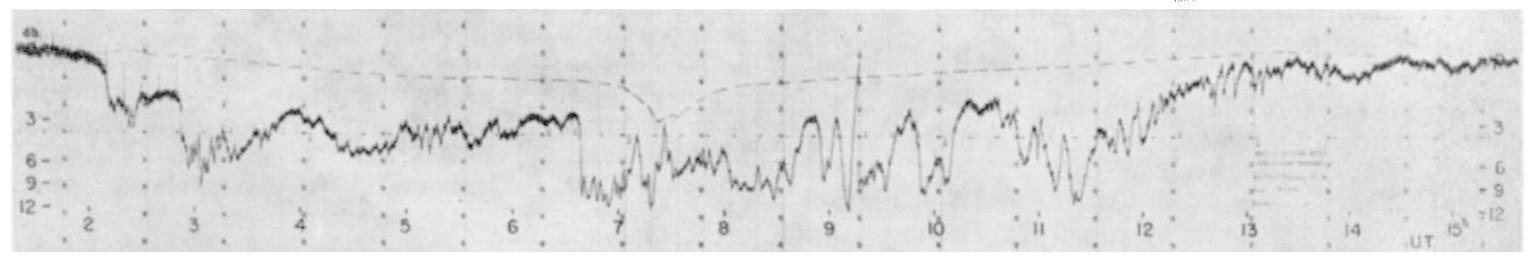

Fig. 1. Absorption of 18-Mc./s. cosmic noise during the auroral display of February 11, 1958, plotted against Tniversal Time 